\title{
Meningeal Solitary Fibrous Tumors with Delayed Extracranial Metastasis
}

\author{
Nayoung $\mathrm{Han}^{1 *} \cdot$ Hannah $\mathrm{Kim}^{1 *}$ \\ Soo Kee $\mathrm{Min}^{2}$ · Sun-Ha Paek ${ }^{3}$ \\ Chul-Kee Park ${ }^{3} \cdot$ Seung-Hong Choi ${ }^{4}$ \\ U-Ri Chae ${ }^{5}$ Sung-Hye Park ${ }^{1,6}$ \\ 'Department of Pathology, Seoul National \\ University College of Medicine, Seoul; \\ 2Department of Pathology, Hallym University \\ Sacred Heart Hospital, Hallym University \\ College of Medicine, Anyang; Departments of \\ ${ }^{3}$ Neurosurgery and ${ }^{4}$ Radiology, Seoul National \\ University College of Medicine, Seoul; \\ ${ }^{5}$ Department of Industrial Engineering, Ajou \\ University, Suwon; ${ }^{6}$ Neurosicence Institute, Seoul \\ National University College of Medicine, Seoul, \\ Korea
}

Received: August 3, 2015

Revised: October 11, 2015

Accepted: October 30, 2015

\section{Corresponding Author}

Sung-Hye Park, MD

Department of Pathology, Seoul National University

College of Medicine, 103 Daehak-ro, Jongno-gu,

Seoul 03080, Korea

Tel: $+82-2-740-8278$

Fax: $+82-2-765-5600$

E-mail: shparknp@snu.ac.kr

${ }^{*}$ Nayoug Han and Hannah Kim contributed equally to this work.

\begin{abstract}
Background: The term solitary fibrous tumor (SFT) is preferred over meningeal hemangiopericytoma (HPC), because NAB2-STAT6 gene fusion has been observed in both intracranial and extracranial HPCs. HPCs are now considered cellular variants of SFTs. Methods: This study analyzes 19 patients with STAT6-confirmed SFTs, who were followed for over 11 years in a single institution. Ten patients $(10 / 19,56.2 \%)$ had extracranial metastases (metastatic group), while the remainder (9/19) did not (non-metastatic group). These two groups were compared clinicopathologically. Results: In the metastatic group, the primary metastatic sites were the lungs $(n=6)$, bone $(n=4)$, and liver $(n=3)$. There was a mean lag time of 14.2 years between the diagnosis of the initial meningeal tumor to that of systemic metastasis. The median age at initial tumor onset was 37.1 years in the metastatic group and 52.5 in the non-metastatic group. The 10-year survival rates of the metastatic- and non-metastatic groups were $100 \%$ and $33 \%$, respectively. The significant prognostic factors for poor outcomes on univariate analysis included advanced age $(\geq$ 45 years) and large initial tumor size $(\geq 5 \mathrm{~cm})$. In contrast, the patients with higher tumor grade, high mitotic rate ( $\geq 5 / 10$ high-power fields), high Ki-67 index ( $\geq 5 \%)$, and the presence of necrosis or CD34 positivity showed tendency of poor prognosis but these parameters were not statistically significant poor prognostic markers. Conclusions: Among patients with SFTs, younger patients $(<$ 45 years) experienced longer survival times and paradoxically had more frequent extracranial metastases after long latent periods than did older patients. Therefore, young patients with SFTs require careful surveillance and follow-up for early detection of systemic metastases.
\end{abstract}

Key Words: Central nervous system; Hemangiopericytoma; Neoplasm metastases; NAB2-STAT6 gene fusion; Solitary fibrous tumors
Solitary fibrous tumors (SFTs), first described in 1931 by Klemperer and Coleman, ${ }^{1}$ are rare spindle-cell mesenchymal tumors that often arise in the pleural cavity. However, SFTs can arise in the extrapleural sites such as thoracic wall, mediastinum, pericardium, retroperitoneum, and abdominal cavity. They can also occur in the subcutaneous and deep soft tissues of the extremities and extracompartmentally in the head and neck. ${ }^{1,2}$ More than 100 cases of intracranial SFTs have been reported to date. However, the exact incidence of intracranial SFTs is unknown. The current consensus is that extracranial hemangiopericytomas (HPCs) and SFTs are synonymous. Such tumors should be called SFTs (rather than HPCs), because HPCs do not originate from or differentiate into pericytes. ${ }^{2}$ The prototypical pericytic neoplasms are myopericytomas and sinonasal HPCs. ${ }^{2}$ However, in the central nervous system (CNS), HPCs and SFTs exhibit distinct biological behavior, and are therefore considered separate entities. For instance, intracranial HPCs tend to be aggressive, while intracranial SFTs are typically relatively benign. ${ }^{3}$

Recently, NAB2-STAT6 gene fusions have been identified using whole-exome sequencing in intracranial and extracranial soft tissue HPCs and SFTs. ${ }^{4}$ Therefore, the use of a single, unifying term to describe intracranial SFTs is preferred than HPCs with the HPC morphology implies a "cellular and aggressive variant," and SFT morphology a "fibrous and benign variant" of the same disease. $^{5}$

Meningeal HPCs commonly occur around the same age as do meningiomas (range, 26 to 73 years; mean, 48 years). However, compared to meningiomas, HPCs are more common in men 
than in women. ${ }^{6}$ According to Pistolesi et al.'s report, the mean time to the first local recurrence and to the appearance of extraneural metastases of meningeal HPCs were 3.9 years and 8.3 years, respectively. The 10 -year survival rate was $83.9 \%{ }^{6}$

This study was designed to clarify the clinicopathological characteristics of metastatic and non-metastatic meningeal SFTs. We analyzed meningeal SFTs (cellular variant) displaying extracranial dissemination, and compared them with non-metastatic meningeal SFTs. Patients with metastatic meningeal SFTs (metastatic group) were significantly younger than were those with non-metastatic tumors (non-metastatic group). Interestingly, the metastatic group exhibited better long-term survival than did the non-metastatic group.

\section{MATERIALS AND METHODS}

All cases of meningeal SFTs/HPCs between January 1995 and
January 2013 were retrieved from the pathology archives at the Seoul National University Hospital (SNUH). We selected cases that matched the search term "solitary fibrous tumor" or "hemangiopericytoma," and 48 cases of meningeal HPCs/SFTs were selected from this 18 -year period. There was no sex predominance. The mean patient age was 48.1 years (range, 21 to 77 years). We selected 19 cases of pathologically and STAT6 immunohistochemically confirmed meningeal SFTs that were followed up for more than 11 years. Ten cases presented with systematic extracranial metastases (metastatic group), while nine had no extracranial metastases (non-metastatic group). The medical and pathological records of the19 cases are summarized in Table 1.

Two pathologists (N.H and S.-H.P) performed all of the pathological reviews. A Ventana autostainer was used for immunohistochemical staining, according to the manufacturer guidelines. Primary antibodies against STAT6 (1:1,000, clone Sc-621, Santa Cruz Biotechnology, Santa Cruz, CA, USA), CD34 (1:200,

Table 1. Summary of the patients with and without meningeal SFT/HPC and extracranial metastasis, treated in Seoul National University Hospital (SNUH)

\begin{tabular}{|c|c|c|c|c|c|c|c|c|c|c|c|}
\hline No. & $\begin{array}{l}\text { Age at first } \\
\text { diagnosis } \\
(y r)\end{array}$ & Sex & Dx & Site & $\begin{array}{c}\text { Site of } \\
\text { metastasis }\end{array}$ & Symptoms & $\begin{array}{l}\text { Initial } \\
\text { Tx }\end{array}$ & Adjuvant Tx & $\begin{array}{l}\text { Local } \\
\text { recurrence }\end{array}$ & $\begin{array}{l}\text { Time for } \\
\text { metastasis } \\
(y r)\end{array}$ & $\begin{array}{c}\text { Mortality } \\
\text { and cause } \\
\text { of death }\end{array}$ \\
\hline 1 & 26 & $\mathrm{~F}$ & HPC & Cerebellum & Bone, lung & Headache & GTR & RT & No & 11.3 & DBD \\
\hline 2 & 56 & $\mathrm{~F}$ & HPC & Rt. frontal & Lung & L/E weakness & GTR & CT (VIP \#1) & Yes & 11.0 & DBD \\
\hline 3 & 24 & $\mathrm{~F}$ & HPC & Lt. occipital & Liver & Headache & GTR & $\begin{array}{l}\text { GKS, CT } \\
\text { (ifosfamide \#1) }\end{array}$ & Yes & 17.6 & Alive \\
\hline 4 & 36 & M & $\mathrm{HPC}$ & Lt. sagittal & Bone & Headache & STR & $\mathrm{RT}$ & Yes & 11.7 & Alive \\
\hline 5 & 52 & F & HPC & Rt. temporal & Bone, EAC & Headache & GTR & GKS, RT & Yes & 5.7 & DBD \\
\hline 6 & 30 & M & HPC & Rt. CPA & $\begin{array}{l}\text { Lung, liver, } \\
\text { orbit, spinal } \\
\text { cords }\end{array}$ & Dizziness & GTR & Cyberknife & Yes & 5.6 & DBD \\
\hline 7 & 32 & M & HPC & Lt. T-P-O & Lung & Headache & GTR & GKS & Yes & 22.8 & Alive \\
\hline 8 & 44 & M & HPC & Cerebellum & Bone, lung & $\begin{array}{l}\text { Headache, } \\
\text { L/E weakness }\end{array}$ & GTR & GKS, RT & Yes & 24.2 & DBD \\
\hline 9 & 29 & $\mathrm{~F}$ & HPC & Lt. frontal & Lung & Incidental & GTR & None & No & 22.1 & Alive \\
\hline 10 & 43 & $\mathrm{~F}$ & SFT & Rt. F-T & $\begin{array}{l}\text { Liver, breast, } \\
\text { lung }\end{array}$ & Double vision & GTR & Preop-RT & No & 10.3 & Alive \\
\hline 11 & 73 & $\mathrm{~F}$ & HPC & $\begin{array}{l}\text { Rt. fronto- } \\
\text { parietal }\end{array}$ & NA & L/E weakness & GTR & Re-GTR & Yes & NA & Alive \\
\hline 12 & 47 & $\mathrm{~F}$ & HPC & Lt. frontal & NA & $\begin{array}{l}\text { Thyroid cancer } \\
\text { work up }\end{array}$ & GTR & None & Unknown & NA & Death \\
\hline 13 & 34 & M & $\mathrm{HPC}$ & Cerebellum & NA & Headache & GTR & $\mathrm{RT}$ & No & NA & Alive \\
\hline 14 & 61 & $\mathrm{~F}$ & $\mathrm{HPC}$ & Lt. occipital & NA & Headache & GTR & GKS, RT & Yes & NA & DBD \\
\hline 15 & 62 & $\mathrm{~F}$ & HPC & Medial parietal & NA & L/E weakness & GTR & GKS, RT & Yes & NA & DBD \\
\hline 16 & 58 & $\mathrm{~F}$ & HPC & Parasagittal & NA & Unknown & GTR & Unknown & Unknown & NA & Death \\
\hline 17 & 50 & M & HPC & Lt. parietal & NA & Unknown & GTR & Unknown & Unknown & NA & Death \\
\hline 18 & 53 & F & SFT & Rt. parietal & NA & $\begin{array}{l}\text { Visual } \\
\text { disturbance }\end{array}$ & GTR & None & No & NA & Alive \\
\hline 19 & 35 & $\mathrm{~F}$ & $\mathrm{HPC}$ & Falx & NA & Headache & GTR & None & Unknown & NA & Death \\
\hline
\end{tabular}

SFT, solitary fibrous tumor; HPC, hemangiopericytoma; Tx, therapy; F, female; HPC, hemangiopericytoma; GTR, gross total resection; RT, radiotherapy; DBD, death by disease; Rt., right; L/E, lower extremity; CT, chemotherapy; VIP, VP-16, ifosfamide, cisplatin; Lt., left; GKS, gamma knife surgery; M, male; STR, subtotal resection; EAC, external auditory canal; CPA, cerebellopontine angle; T-P-O, temporoparietooccipital lobe; SFT, solitary fibrous tumor; F-T, frontotemporal; NA, not applicable. 
Dako, Glostrup, Denmark), epithelial membrane antigen (EMA; Dako, 1:200), and MIB-1 (Ki-67) (Dako, 1:1,000) were used. A verified SFT or antibody-specific known positive control was used as a positive control. The primary antibodies were omitted in the negative control. For STAT6, robust nuclear positivity in $>90 \%$ of the tumor cells was considered positive. For CD34, cytoplasmic staining in $>10 \%$, and for EMA, membranous staining in $>10 \%$ of the tumor cells were considered positive. A nuclear algorithm of the Aperio ScanScope image analysis program (Aperio Technologies, Vista, CA, USA) was used to calculate the nuclear positivity in hot spots for the Ki-67 labeling index.

Statistical analyses were performed using Fisher exact tests. Kaplan-Meier survival analysis was performed to compare overall survival according to the patients' age, tumor grade, mitoses, necrosis, Ki-67 labeling index, CD34 expression, and the occurrence of systemic metastasis. All statistical analyses were carried out using SPSS ver. 22 (IBM Co., Armonk, NY, USA).

The mean patient ages in the metastatic and non-metastatic groups were 37.1 years (range, 26.1 to 55.9 years) and 52.5 years (range, 34 to 76.2 years), respectively (Table 2). The mean followup periods for the metastatic and non-metastatic groups were 215 months (range, 124 to 351 months) and 99 months (range, 33 to 174 months), respectively. This study was designed to clarify the clinicopathological characteristics of metastatic and nonmetastatic meningeal SFTs. In order to identify prognostic factors, several parameters were compared between the two groups, including Ki-67 labeling indices, mitotic rates, initial meningeal tumor size, and the presence or absence of necrosis.

This study abides by the World Medical Association Declaration of Helsinki recommendations. It was approved by the Institutional Review Board of Seoul National University Hospital (IRB No. 1501-046-639).

\section{RESULTS}

Six of the ten patients in the metastatic group were women, and four were men. There was an average age of 37.1 years (range, 24 to 56 years). The initial symptoms included headache (6 patients), lower extremity weakness (2), double vision (1), and dizziness (1). In one patient, the diagnosis was an incidental finding. The disease originated in the supratentorial region in eight patients, and in the posterior fossa in the other two. There was no intracranial predilection site. Nine patients underwent gross total resections (GTR) of their tumors, while one (case 4) had a partial resection.

Radiologically, the tumors were large, well-enhanced, solid, and cystic masses attached to the dura. Typically, they had intratumoral vessels, and exhibited a severe mass effect. One patient (case 10) underwent preoperative radiotherapy (RT; 54 Gy) due to the tumor's excessive size $(6.5 \times 5.8 \times 5.5 \mathrm{~cm})($ Fig. $1 \mathrm{~A})$. The solid and cystic tumor decreased in size, as evidenced by a postRT brain magnetic resonance imaging (Fig. 1B). Nine of the 10 patients in this group were treated with adjuvant therapies following GTR (Table 1). The types of adjuvant therapy included gamma knife surgery (GKS) in four patients, RT in three, Cyberknife $\mathrm{RT}$ in one, and chemotherapy in one.

In the non-metastatic group, all patients underwent GTR. During the follow-up period, one patient received RT and two received RT + GKS (Table 1).

In the metastatic group, seven patients (70\%) had extracranial metastases after local recurrence was detected. In the remaining three, the tumors metastasized without local recurrence. Common extracranial metastatic sites included the lungs (6 patients), bone (4), and liver (3). Other less frequent metastatic sites included the breast (1 patient), orbit (1), and external auditory canal (1). When the disease was metastatic to the bone, common sites included the vertebrae, femur, and ischium. When the tumor invaded the lungs and bone, multiple metastases were observed. In contrast, metastatic foci in the liver and breast were usually single masses (Fig. 2). However, lymph node enlargement or lymph node metastases were not present. The average time from the initial diagnosis of a meningeal tumor to systemic metastasis was 14.2 years (range, 5.6 to 24.2 years). The findings of these 10 cases with systemic metastasis are summarized in Table 1.

Table 2. The differences between the meningeal SFTs according to the presence or absence of systemic metastases

\begin{tabular}{lcc}
\hline Item & SFT with systemic metastases (metastatic group, $\mathrm{n}=10)$ & SFT without systemic metastases (non-metastatic group, $\mathrm{n}=9$ ) \\
\hline Age $(\mathrm{yr})$ & $37(24.1-55.9)$ & $51(33.9-76.4)$ \\
Median mitotic rate & $7 / 10 \mathrm{HPF}(1-27)$ & $7 / 10 \mathrm{HPF}(1-26)$ \\
Median Ki-67 labeling index (\%) & 5.0 & 6.0 \\
Median tumor size (cm) & $3.5(2.2-6.0)$ & $5.0(1.5-7.5)$ \\
Median survival & $202 \mathrm{mo}(16.8 \mathrm{yr})$ & $56 \mathrm{mo}(4.7 \mathrm{yr})$
\end{tabular}

SFT, solitary fibrous tumor; HPF, high-power field. 

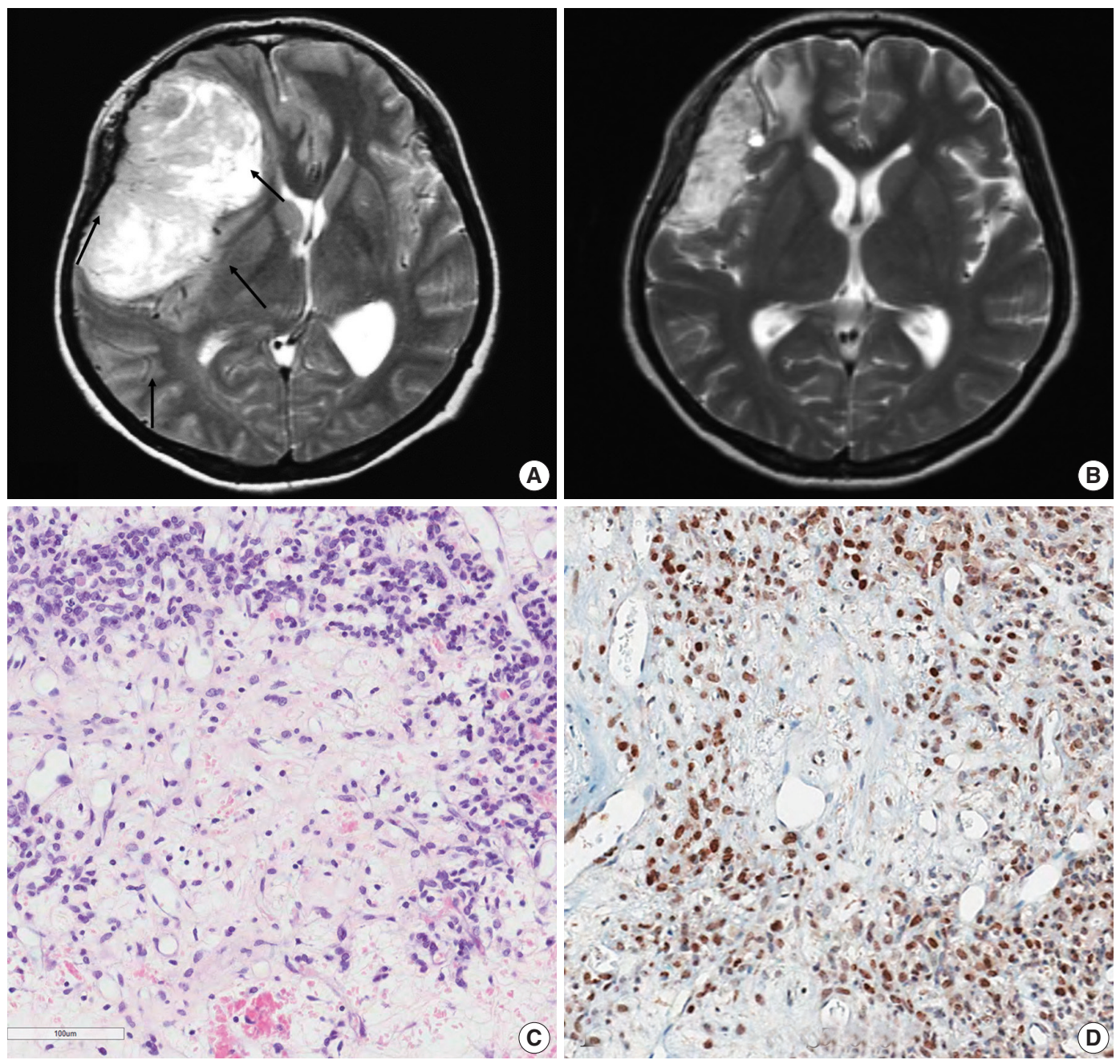

Fig. 1. Representative brain magnetic resonance images and histopathology of the primary brain tumor from case 10. (A) Prior to radiation therapy. A large, solid and cystic mass is observed in the right frontoparietal lobe with strong enhancement. There was severe mass effect. Intratumoral vessels were identified. (B) After preoperative radiation therapy. The tumor decreased in the size after radiation therapy. (C) The tumor demonstrates low cellular and edematous areas with staghorn-shaped vasculature. The tumor cells may have been ablated by radiotherapy. (D) Aberrant STAT6 nuclear positivity is observed in the primary brain tumor.

Histopathologically, the initial meningeal and metastatic tumors are identical to cellular SFTs (previously called intracranial HPC-like tumors of a cellular nature) (Figs. 1 and 2 are from the same patient, case 10). Interestingly, multinucleated osteoclasttype giant cells (OGCs) were widely present in one metastatic tumor to the liver (Fig. 2C). There was necrosis in 30\% and 28\% of tumors with and without systemic metastases, respectively. All of the tumors exhibited robust, but heterogeneous STAT6 immunopositivity. In most cases, the STAT6 immunostaining was stronger at the periphery of the tumor or in the perivascular areas (Figs. 1, 2). However, $40 \%$ of systemically metastatic tumors had positive CD34 immunostaining, while $85 \%$ of nonmetastatic tumors showed at least focal CD34 positivity. EMA immunostaining was focally positive in two cases. The mean
Ki-67 indices were $8.7 \%$ and $8.8 \%$ (1.0\% to $27.3 \%$ ) in the tumors of metastatic and non-metastatic groups, respectively. There were no differences in the Ki-67 labeling indices, mitoses, necrosis, or tumor size based on the presence or absence of systemic metastasis (Table 2).

Ultrastructural studies were carried out in three patients from the metastatic group and seven from the non-metastatic group. There were no differences between the two groups. Ultrastructural examination revealed sheets of oval to elongated cells around the small capillaries. The tumor nuclei were oval or indented in appearance. The nuclear chromatin was fine and granular, with skein-like nucleoli. The individual tumor cells were surrounded by thick, electron-dense external laminar material (Fig. 2F).

Over the 10-29-year (average, 17.2 years) follow-up period, 

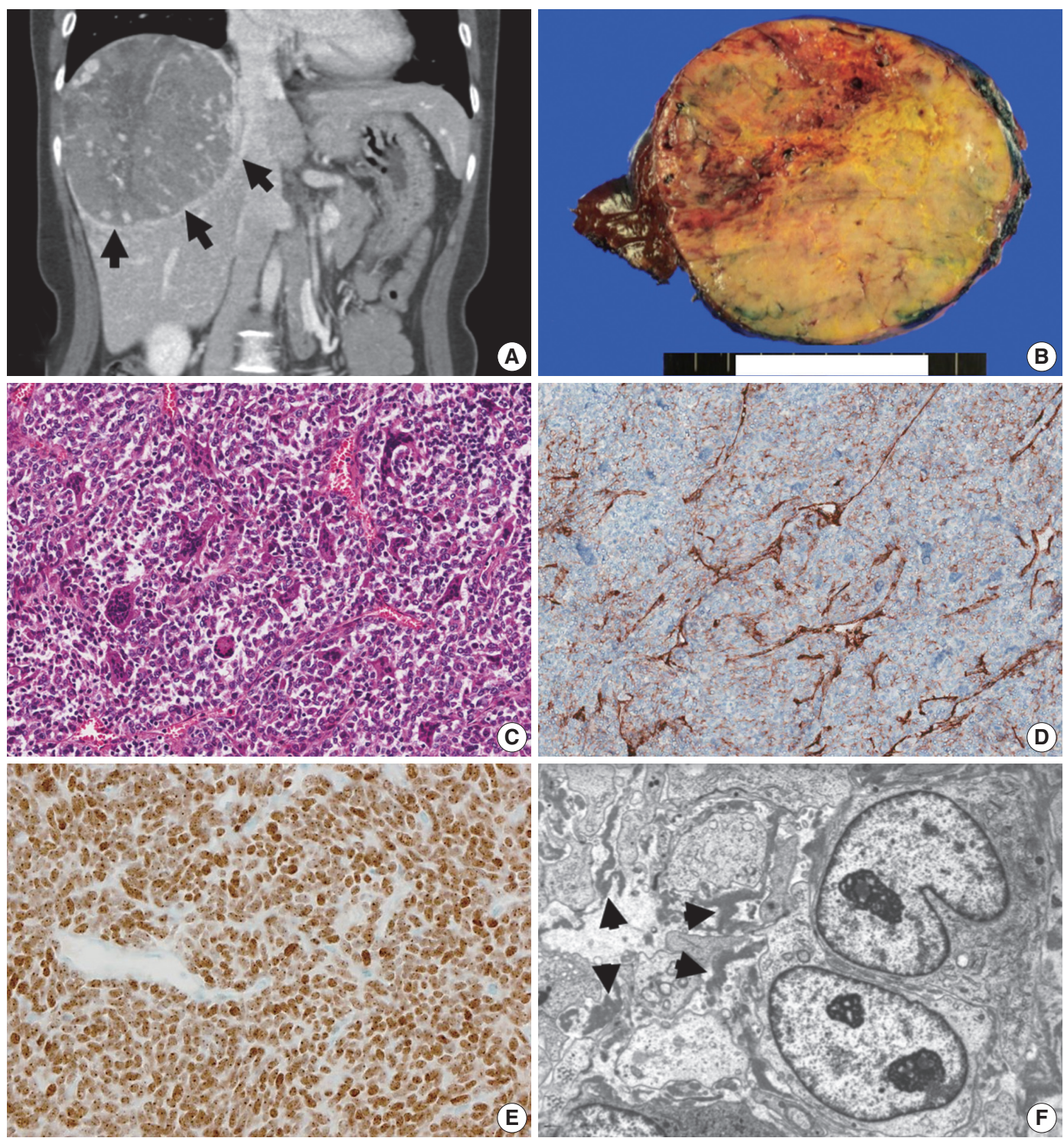

Fig. 2. Computed tomography (CT) and pathology of a metastatic solitary fibrous tumor from case 10. (A) Liver CT scan showing a large, 12$\mathrm{cm}$ soft tissue mass in the right lobe with prominent feeding vessels. (B) The cut surface of a hepatic tumor specimen showing a well-circumscribed, yellowish-tan-colored, solid tumor with multifocal necrosis, and hemorrhage with dilated vessels. (C) Microscopic examination of the liver mass reveals small, discohesive tumor cells with oval nuclei and fine chromatin. Staghorn-shaped hyalinized vessels and osteoclast-like multinucleated giant cells are identified. (D) Tumor cells are focally positive for CD34 on immunohistochemical staining. (E) Aberrant STAT6 nuclear positivity is observed in the metastatic tumor cells in the liver. (F) Ultrastructurally, the tumor is composed of sheets of oval to elongate cells around small capillaries. The tumor cells have nuclei with an oval or indented appearance, and fine, granular chromatin. Skeinlike nuclei are also present. The individual tumor cells are surrounded by a thick, electron-dense, amorphous, external laminar material (arrowheads; uranyl acetate and lead citrate, $\times 5,000$ ).

five patients $(50 \%)$ in the metastatic group died of their disease. The 10 -year survival rate was $100 \%$, but by 15 years, this rate dropped to $60 \%$. In the non-metastatic group, the 10 -year survival rate was $33 \%$. This rate was sustained for 14.5 years of follow-up.

Paradoxically, the Kaplan-Meier survival analysis revealed that patients with metastatic disease had significantly better over- all survival times than did those without systemic metastases (p $=.04)$ (Fig. 3). The mean survival of patients with systemic metastases was $215 \pm 79$ months, and that of patients without systemic metastases was $99 \pm 47$ months. The median survival time was 202 months and 99 months for patients with and without systemic metastases, respectively.

Both univariate and multivariate analyses revealed that an 

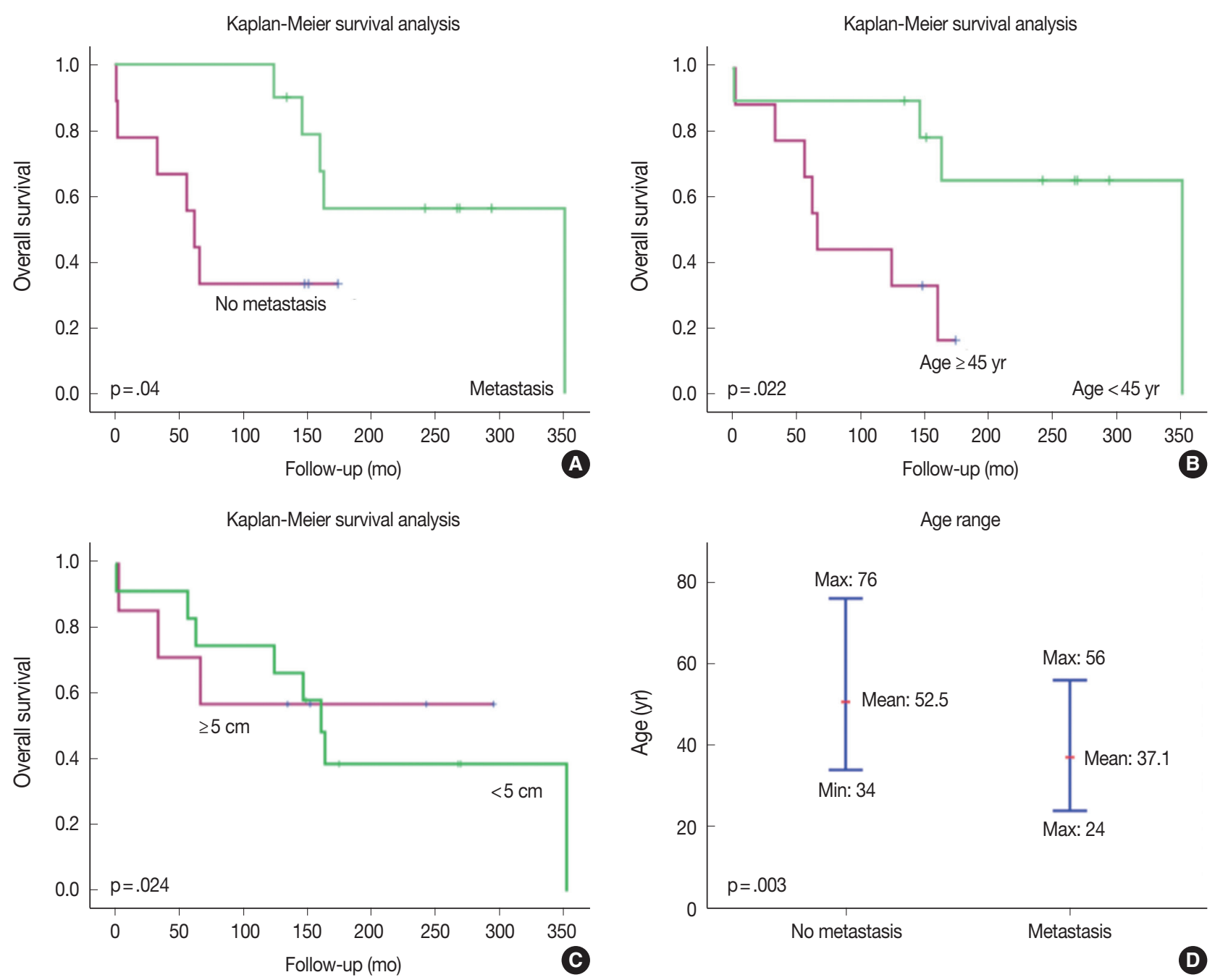

Fig. 3. Kaplan-Meier survival curves of patients with solitary fibrous tumors according to the presence or absence of systemic metastases. (A) Paradoxically, the patients with systemic metastases had better prognosis than did those without metastasis. Metastases usually arose approximately 10 years after the initial tumor development. Therefore, only longer survivors developed systemic metastases. (B) Younger patients lived longer and had better survival. The age cut-off to demonstrate a meaningful survival difference is 45 years. (C) Patients with an initial tumor size $>5 \mathrm{~cm}$ have poorer survival than did those with smaller tumors. (D) The age of tumor onset is significantly younger in patients with systemic metastases than it is in patients without metastases. The median ages of onset in patients with and without systemic metastases are 37.1 years and 52.5 years, respectively.

age of onset $>45$ years and an initial tumor size $>5 \mathrm{~cm}$ were significant markers of poor prognosis. There was no prognostic significance of increased mitoses ( $\geq 5 / 10$ high-power fields), high $\mathrm{Ki}-67$ index ( $\geq 5 \%$ ), necrosis, high tumor grade, or the absence of CD34 expression on univariate and multivariate analyses. However, these factors did suggest a poor prognosis on multivariate analysis (Table 3).

\section{DISCUSSION}

A SFT is an uncommon type of spindle-cell mesenchymal tumor. It is most commonly found in the visceral pleura, but also has been reported in virtually all extrapleural soft tissues. ${ }^{5,8}$ Recently, SFTs and HPCs in soft tissue have been reclassified as a single entity. ${ }^{3}$ Gengler and Guillou' introduced the concept of an SFT spectrum. They recommend that a conventional SFT is designated as a fibrous variant of an SFT, while a conventional HPC is considered a cellular variant of an SFT.

Regardless of where they develop in the CNS, there is morphological, immunohistochemical, and genetic overlap between SFTs and HPCs. ${ }^{10}$ However, in the CNS, HPCs are still considered separate entities from SFTs. ${ }^{11}$ The so-called meningeal SFTs have generally been regarded as indolent, non-aggressive tumors. ${ }^{3,12}$ Conversely, meningeal HPCs have a high tendency to 
Table 3. The results of univariate and multivariate analysis

\begin{tabular}{lcccc}
\hline \multirow{2}{*}{ Variable } & Univariate analysis & & \multicolumn{2}{c}{ Multivariate analysis } \\
\cline { 3 - 5 } & $p$-value & Hazard ratio & $95 \% \mathrm{Cl}$ & $\mathrm{p}$-value \\
\hline Age $(<45$ yr vs $\geq 45 \mathrm{yr})$ & .022 & 0.172 & $0.009-3.277$ & .241 \\
Systemic metastasis & .040 & 0.126 & $0.01-1.675$ & .117 \\
Tumor size $(<5 \mathrm{~cm}$ vs $\geq 5 \mathrm{~cm})$ & .377 & 2.815 & $0.364-21.796$ & .322 \\
Necrosis & .352 & 1.28 & $0.225-7.291$ & .781 \\
Mitoses $(<5 / 10 H P F$ vs $\geq 5 / 10 H P F)$ or grade & .603 & 6.602 & $0.12-362.385$ & .356 \\
Ki-67 labeling index $(<5 / 10 H P F$ vs $\geq 5 / 10 H P F)$ & .377 & 0.261 & $0.009-7.773$ & .438 \\
CD34 (positive vs negative) & .983 & 0.246 & $0.26-2.319$ & .22 \\
\hline
\end{tabular}

Cl, confidence interval; HPF, high-power field.

Table 4. Summary of the patients with meningeal SFT/HPC with extracranial metastasis in the literature

\begin{tabular}{|c|c|c|c|c|}
\hline Reference & Age $(y r) / S e x$ & Diagnosis & Metastatic location & Time for metastasis (yr) \\
\hline $\mathrm{Ng}$ et al. $(2000)^{14}$ & $55 / F$ & $\mathrm{SFT}$ & Lung and neck & 9 \\
\hline \multirow[t]{2}{*}{ Someya et al. (2001) } & $42 / F$ & HPC & Bone, lung, and liver & 12 \\
\hline & $37 / F$ & $\mathrm{HPC}$ & Bone & 7 \\
\hline \multirow[t]{3}{*}{ Dufour et al. $(2001)^{16}$} & 23/M & HPC & Bone & 12 \\
\hline & 22/F & $\mathrm{HPC}$ & Extracranial site & 13 \\
\hline & 30/M & $\mathrm{HPC}$ & Extracranial site & 14 \\
\hline Ogawa et al. $(2004)^{13}$ & $44 / F$ & SFT & Lung & 25 \\
\hline Pistolesi et al. (2004) ${ }^{7}$ & $42 / F$ & HPC & Bone, lung and adrenal gland & 13 \\
\hline Chang et al. (2004) ${ }^{17}$ & $43 / F$ & $\mathrm{HPC}$ & C2-C3 vertebrates, lung, liver and kidney & 5 \\
\hline Metellus et al. $(2007)^{10}$ & $34 / \mathrm{M}$ & SFT & Systemic metastasis & 10.5 \\
\hline \multirow[t]{4}{*}{ Hayashi et al. $(2009)^{18}$} & $45 / M$ & $\mathrm{HPC}$ & Extracranial site & NM \\
\hline & 30/M & $\mathrm{HPC}$ & Extracranial site & NM \\
\hline & $47 / F$ & HPC & Extracranial site & NM \\
\hline & $35 / M$ & HPC & Extracranial site & NM \\
\hline Ambrosini-Spaltro and Eusebi (2010) ${ }^{11}$ & $51 / F$ & HPC & Hip & 13 \\
\hline \multirow[t]{4}{*}{ Robinson et al. (2013) ${ }^{20}$} & 40/M & Malignant SFT & Lung & NM \\
\hline & 33/F & Malignant SFT & Kidney & NM \\
\hline & 29/M & Malignant SFT & Pancreas & NM \\
\hline & $32 / \mathrm{M}$ & Malignant SFT & Small bowel & NM \\
\hline
\end{tabular}

SFT, solitary fibrous tumor; HPC, hemangiopericytoma; F, female; M, male; NM, not metastasized.

recur and to metastasize outside of the CNS; therefore, these are classified as World Health Organization grade II or III lesions. ${ }^{12}$ Histopathologically, meningeal HPCs tend to have higher rates of mitosis and a higher Ki-67 index than do HPCs/SFTs in soft tissues. They also stain less intensely for CD34 and B-cell lymphoma 2 (BCL2) proteins. ${ }^{11}$ However, two previous studies reported that several cases of SFTs progressed to HPCs at recurrence. ${ }^{3,10}$ There have also been reports of extracranial metastases of conventional SFTs. ${ }^{13}$

In the English-language literature, we identified 19 reports of meningeal SFTs/HPCs with extracranial metastasis (Table 4). ${ }^{14-18}$ The median age of tumor onset of these cases was 37 years, and there was no sex dominance (male:female =9:10). The median time between the initial tumor development and extracranial metastasis was 14.2 years (range, 5.6 to 24.2 years).

In our series, patients with metastatic meningeal SFTs were a mean of 15 years younger than were those in the non-metastatic group. There was a female sex predominance (male:female = 2:3). Similar to the prior report, the mean time between the initial tumor development and extracranial metastasis was 14.2 years in our study. ${ }^{19}$ Hoshi et al ${ }^{19}$ reported that two cases had metastasized to the bone, 17 and 19 years after resection of primary leptomeningeal HPCs and introduced that median time from primary tumor to the occurrence of systemic metastases was 149 years from literature review. The most common metastatic sites were the lungs, bone, and liver, but rarely, the breast, orbit, and external auditory canal were the metastatic site. There were no lymph node metastases. Therefore, the meningeal SFTs studied here present with very similar clinical features to those previously reported.

Two recent studies found that the NAB2-STAT6 gene fusion was a distinct molecular feature of SFTs and HPCs, in both men- 
ingeal and extracranial tumors. ${ }^{4,20}$ Schweizer $e t$ al. ${ }^{21}$ also found that nuclei stained positive for anti-STAT6 in 35 of 37 meningeal HPCs and in all 25 meningeal SFTs, but not in 87 meningiomas. This provided additional evidence in support of unifying the terms SFT and HPC. Robinson et al. ${ }^{20}$ noted that overexpression of the fusion protein NAB2-STAT6 induces the proliferation of cultured cells and activates early growth response genes. These findings suggest that the NAB2-STAT6 fusion is a driver mutation of $\mathrm{SFT}^{20}$

In this study, strong aberrant nuclear expression of STAT6 was detected in all meningeal and extracranial metastatic SFTs (including the cases previously diagnosed as HPCs). This suggests that these tumors had the same pathogenesis. Verifying nuclear STAT6 immunoexpression or NAB2-STAT6 gene fusion may be useful to confirm the diagnosis, especially when only small biopsies are available for pathology, or in cases that are CD34-negative. Although CD34 is a good marker of SFTs/HPC, its sensitivity is $<100 \%$. Overall, there is $95 \% \mathrm{CD} 34$ positivity in SFTs. ${ }^{22}$ However, the CD34 positivity is lower in meningeal SFTs, as mentioned above. ${ }^{11}$

To our knowledge, this is the first report of prominent OGCs described in an SFT/HPC (case 9). Previously, OGCs have been reported in various malignancies including breast, pancreatic, and gastric carcinomas, ${ }^{23-25}$ gastrointestinal stromal tumors, ${ }^{26}$ clear cell sarcoma-like tumors, ${ }^{27}$ squamous cell carcinoma of the skin ${ }^{28}$ and malignant melanoma. ${ }^{29}$ It is thought that OGCs result from a host reaction to degeneration and necrosis of the tumor. However, it is not well understood why OGCs are so exaggerated in non-histiocytic tumors.

Kaplan-Meier survival analysis revealed that patients with metastatic disease had significantly better survival than did those without systemic metastasis ( $\mathrm{p}=.04$ ) (Fig. 3). The mean age of the metastatic group was younger than that of the non-metastatic group ( 37.1 years vs 52.5 years, respectively), suggesting that the age might be the most important prognostic factor (Fig. 3). Younger patients lived longer, but also had a higher risk of metastasis than did older patients. The Ki-67 labeling index ( $25 \%)$, presence of necrosis, and absence of $\mathrm{CD} 34$ expression were not significant prognostic factors on univariate and multivariate analyses. The statistical insignificance of these latter factors may reflect the small number of cases involved.

In this study, two patients received adjuvant chemotherapy, one patient received a single cycle of VIP (VP-16, ifosfamide, cisplatin) chemotherapy and one received a single cycle of ifosfamide. There is no optimal chemotherapy regimen that has been established for SFTs/HPCs to date. ${ }^{30}$ There are limited case reports describing chemotherapy use. One case report described a patient with meningeal SFTs/HPCs who received two cycles of ICE (ifosfamide, carboplatin, and etoposide) chemotherapy and lived at least 151 months. ${ }^{6}$ Another study reported the relative success of various chemoregimens in 15 patients. ${ }^{31}$ In that study, only two patients were treated with adjuvant chemotherapy, but the treatment was stopped after one cycle in one patient due to a severe adverse effect.

In conclusion, we have reported 10 cases of meningeal SFTs with delayed extracranial metastasis. Systemic metastases developed after a long latent period, a mean of 14.2 years after the initial surgery. Metastases were associated with younger patient age and longer survival. Leptomeningeal SFTs can be divided into two groups by prognosis. The poor prognostic group is characterized by an initially locally aggressive tumor and early death. The better prognostic group is characterized by younger age of tumor onset, successful control of local recurrence, and a life expectancy $>10$ years. However, despite intensive treatment, this group eventually developed delayed systemic metastases. The median survival rates of patients with and without systemic metastases were 202 months and 99 months, respectively. The age of onset was the most important prognostic factor. The best prognostic group was $<45$ years old on Kaplan-Meier survival analysis. Therefore, meningeal SFTs arising in young patients (< 45 years) require careful surveillance for the early detection of systemic metastases, especially because extracranial metastases usually occur after a latency period of $>10$ years.

\section{Conflicts of Interest}

No potential conflict of interest relevant to this article was reported.

\section{Acknowledgments}

This work was supported by the National Research Foundation of Korea (NRF) grant funded by the Korean government (MSIP) (No. 2010-0028631).The authors would like to thank U-ri Chae (Department of Industrial Engineering, Ajou University) for her precise statistical analysis of our data. In addition, the authors are very grateful to Professor Je G. Chi, who passed away last winter (November 26, 2014). He was a great mentor and friend to all of us. This work is dedicated to his memory.

\section{REFERENCES}

1. Klemperer P, Coleman BR. Primary neoplasms of the pleura: a re- 
port of five cases. Am J Ind Med 1992; 22: 1-31.

2. Fletcher CD, Bridege JA, Hogendoorn P, Mertens F. WHO classification of tumours of soft tissue and bone. Lyon: IARC Press, 2013.

3. Tihan T, Viglione M, Rosenblum MK, Olivi A, Burger PC. Solitary fibrous tumors in the central nervous system: a clinicopathologic review of 18 cases and comparison to meningeal hemangiopericytomas. Arch Pathol Lab Med 2003; 127: 432-9.

4. Chmielecki J, Crago AM, Rosenberg M, et al. Whole-exome sequencing identifies a recurrent NAB2-STAT6 fusion in solitary fibrous tumors. Nat Genet 2013; 45: 131-2.

5. Yu HC, Cho BH, Kim YK, Noh SJ, Moon WS. Solitary fibrous tumor of the liver: a case report. Korean J Pathol 2010; 44: 536-9.

6. Park BJ, Kim YI, Hong YK, Jeun SS, Lee KS, Lee YS. Clinical analysis of intracranial hemangiopericytoma. J Korean Neurosurg Soc 2013; 54: 309-16.

7. Pistolesi S, Fontanini G, Barellini L, et al. Meningeal hemangiopericytoma metastatic to the adrenal gland with multiple metastases to bones and lungs: a case report. Tumori 2004; 90: 147-50.

8. Kim NR, Ro JY, Shin KH, Paik HJ, An JS, Ha SY. Solitary fibrous tumor of the conjunctiva with heretofore undescribed pathologic findings. Korean J Pathol 2011; 45: 315-8.

9. Gengler C, Guillou L. Solitary fibrous tumour and haemangiopericytoma: evolution of a concept. Histopathology 2006; 48: 63-74.

10. Metellus P, Bouvier C, Guyotat J, et al. Solitary fibrous tumors of the central nervous system: clinicopathological and therapeutic considerations of 18 cases. Neurosurgery 2007; 60: 715-22.

11. Ambrosini-Spaltro A, Eusebi V. Meningeal hemangiopericytomas and hemangiopericytoma/solitary fibrous tumors of extracranial soft tissues: a comparison. Virchows Arch 2010; 456: 343-54.

12. Fuller $\mathrm{C}$. WHO classification of tumours of the central nervous system, fourth edition. J Neuropathol Exp Neurol 2008; 67: 260.

13. Ogawa K, Tada T, Takahashi S, et al. Malignant solitary fibrous tumor of the meninges. Virchows Arch 2004; 444: 459-64.

14. Ng HK, Choi PC, Wong CW, To KF, Poon WS. Metastatic solitary fibrous tumor of the meninges: case report. J Neurosurg 2000; 93 : 490-3.

15. Someya M, Sakata KI, Oouchi A, Nagakura H, Satoh M, Hareyama $M$. Four cases of meningeal hemangiopericytoma treated with surgery and radiotherapy. Jpn J Clin Oncol 2001; 31: 548-52.

16. Dufour H, Métellus P, Fuentes S, et al. Meningeal hemangiopericytoma: a retrospective study of 21 patients with special review of postoperative external radiotherapy. Neurosurgery 2001; 48: 756-62.

17. Chang CC, Chang YY, Lui CC, Huang CC, Liu JS. Meningeal hemangiopericytoma with delayed multiple distant metastases. J Chin Med Assoc 2004; 67: 527-32.

18. Hayashi $Y$, Uchiyama N, Nakada M, et al. A reevaluation of the pri- mary diagnosis of hemangiopericytoma and the clinical importance of differential diagnosis from solitary fibrous tumor of the central nervous system. Clin Neurol Neurosurg 2009; 111: 34-8.

19. Hoshi M, Araki N, Naka N, et al. Bone metastasis of intracranial meningeal hemangiopericytoma. Int J Clin Oncol 2005; 10: 208-13.

20. Robinson DR, Wu YM, Kalyana-Sundaram S, et al. Identification of recurrent NAB2-STAT6 gene fusions in solitary fibrous tumor by integrative sequencing. Nat Genet 2013; 45: 180-5.

21. Schweizer L, Koelsche C, Sahm F, et al. Meningeal hemangiopericytoma and solitary fibrous tumors carry the NAB2-STAT6 fusion and can be diagnosed by nuclear expression of STAT6 protein. Acta Neuropathol 2013; 125: 651-8.

22. Fisher C. Immunohistochemistry in diagnosis of soft tissue tumours. Histopathology 2011; 58: 1001-12.

23. Tavassoéli FA, Devilee P. World Health Organization classification of tumours: pathology and genetics of tumours of the breast and female genital organs. Lyon: IARC Press, 2003.

24. Sakai Y, Kupelioglu AA, Yanagisawa A, et al. Origin of giant cells in osteoclast-like giant cell tumors of the pancreas. Hum Pathol 2000; 31: 1223-9.

25. Ushiku T, Shinozaki A, Uozaki H, et al. Gastric carcinoma with osteoclast-like giant cells: lymphoepithelioma-like carcinoma with Epstein-Barr virus infection is the predominant type. Pathol Int 2010; 60: 551-8.

26. Insabato L, Di Vizio D, Ciancia G, Pettinato G, Tornillo L, Terracciano L. Malignant gastrointestinal leiomyosarcoma and gastrointestinal stromal tumor with prominent osteoclast-like giant cells. Arch Pathol Lab Med 2004; 128: 440-3.

27. Friedrichs N, Testi MA, Moiraghi L, et al. Clear cell sarcoma-like tumor with osteoclast-like giant cells in the small bowel: further evidence for a new tumor entity. Int J Surg Pathol 2005; 13: 313-8.

28. Wooff J, Werner D, Murphy J, Walsh N. Osteoclast-like giant cell reaction associated with cutaneous squamous cell carcinoma: a report of 2 cases and review of the literature. Am J Dermatopathol 2009; 31: 282-7.

29. Al-Brahim N, Salama S. Malignant melanoma with osteoclast-like giant cells: an unusual host response: immunohistochemical and ultrastructural study of three cases and literature review. Am J Dermatopathol 2005; 27: 126-9.

30. Park MS, Patel SR, Ludwig JA, et al. Activity of temozolomide and bevacizumab in the treatment of locally advanced, recurrent, and metastatic hemangiopericytoma and malignant solitary fibrous tumor. Cancer 2011; 117: 4939-47.

31. Chamberlain MC, Glantz MJ. Sequential salvage chemotherapy for recurrent intracranial hemangiopericytoma. Neurosurgery 2008; 63: 720-6. 